\title{
Effect of Near-Wall Componental Modification of Turbulence on its Statistical Properties
}

\author{
Bettina Frohnapfel, Yosuke Hasegawa and Nobuhide Kasagi
}

\begin{abstract}
Turbulence control techniques are of great economical and ecological interest. In the present work a fundamental study is carried out in which body forces are introduced in the near-wall region of a turbulent channel flow and thus modify the near-wall behavior. It is investigated how these forces, which selectively act on one of the velocity components, modify near-wall turbulence and its statistical properties with the goal to extract properties that can directly be linked to the skin friction drag. The alignment between the principal axis of the Reynolds stress tensor and the mean flow direction is identified as an interesting quantity in this respect.
\end{abstract}

\section{Procedure}

We carry out direct numerical simulations of a fully developed channel flow with a constant flow rate. The bulk Reynolds number based on the channel height is $R e_{b}=4460$ and the corresponding friction Reynolds number for the uncontrolled case is given by $R e_{\tau}=150$. As a control input for modification of the near-wall velocity fluctuation we consider a feedback body force, $b f_{j}$, which is introduced into the Navier-Stokes equations. The modified momentum equations $(j=1,2,3)$ thus read:

$$
\frac{\partial u_{j}^{+}}{\partial t^{+}}+u_{i}^{+} \frac{\partial u_{j}^{+}}{\partial x_{i}^{+}}=-\frac{\partial p^{+}}{\partial x_{j}^{+}}+\frac{\partial^{2} u_{j}^{+}}{\partial x_{i}^{+} \partial x_{i}^{+}}+\underbrace{\frac{f\left(x_{2}^{+}\right)}{\Phi^{+}}\left(u_{j}^{+}-\overline{U_{j}^{+}}\right)}_{b f_{j}^{+}},
$$

Bettina Frohnapfel

Center of Smart Interfaces, Technical University of Darmstadt, Petersenstr. 32, 64287 Darmstadt, Germany, e-mail: frohnapfel@ csi.tu-darmstadt.de

Yosuke Hasegawa, Nobuhide Kasagi

Department of Mechanical Engineering, The University of Tokyo, 7-3-1 Hongo, Bunkyo-ku, Tokyo 113-8656, Japan, e-mail: hasegawa / kasagi@thtlab.t.u-tokyo.ac.jp 
where the subscript ()$^{+}$indicates normalization with the kinematic viscosity, $v$, and the wall shear velocity, $u_{\tau}$, of the uncontrolled channel flow. The velocity components in streamwise, wall-normal and spanwise direction are given by $u_{1}, u_{2}, u_{3}$ and $u, v, w$, respectively and the decomposition into mean and fluctuating components is defined as: $u_{j}=\overline{U_{j}}+u_{j}^{\prime}$. The last term on the right hand side of equation (1) represents the body force which is proportional to the corresponding velocity fluctuation. The relaxation time constant, $\Phi^{+}$, determines the strength and type of the forcing, and $f\left(x_{2}^{+}\right)$is a step function which determines the region where the forcing is applied on top and bottom wall: $f\left(x_{2}^{+} \leq y_{f}^{+}\right)=1, f\left(x_{2}^{+}>y_{f}^{+}\right)=0$. The control input can thus be varied by modifying the relaxation time constant, $\Phi^{+}$, and the forcing layer thickness, $y_{f}^{+}$. For positive values of $\Phi^{+}$the introduced body force acts to enhance the velocity fluctuations in the corresponding direction; for negative values of $\Phi^{+}$the introduced body force acts as a damping force. In the present study the focus is placed on the influence of the forcing orientation. Therefore, the forcing layer thickness is set to a constant value of $y_{f}^{+}=10$.

\section{Results and Discussion}

Table 1 shows the obtained drag reduction for different exemplary test cases in which a body force according to equation (1) is introduced within the forcing layer. The results show that damping of the wall-normal velocity fluctuation, $v^{\prime}$, and the spanwise fluctuation, $w^{\prime}$, leads to drag reduction $(D R)$. The $D R$ obtained with a fixed value of $\Phi^{+}$is higher for $w$-forcing. This result is not surprising since the body force depends on the strength of the velocity fluctuation itself and similar results have been reported by [5] who employed an exponentially decaying damping force in the near-wall region. With increasing strength of the $w^{\prime}$-damping force, the theoretical maximum of $D R$ (Iwamoto et al., 2005) is realized for $\left(\Phi^{+}\right)^{-1}<-1[1]$.

Table 1 Drag reduction (DR) obtained for channel flows in which different forcing is applied within a forcing layer thickness of $y_{f}^{+}=10$. The forcing strength is given by $\left(\Phi^{+}\right)^{-1}$.

\begin{tabular}{llrr}
\hline forcing & $b f_{j}$ & $\left(\Phi^{+}\right)^{-1}$ & DR \\
\hline u'-damping & $b f_{1}<0$ & $-1 / 15$ & $-7 \%$ \\
v'-damping & $b f_{2}<0$ & $-10 / 15$ & $20 \%$ \\
w'-damping & $b f_{3}<0$ & $-10 / 15$ & $42 \%$ \\
u'-increase & $b f_{1}>0$ & $2 / 150$ & $2 \%$ \\
(weak) & & & \\
u'-increase & $b f_{1}>0$ & $5 / 150$ & $-8 \%$ \\
(strong) & & & \\
v'-increase & $b f_{2}>0$ & $10 / 15$ & $-10 \%$ \\
w'-increase & $b f_{3}>0$ & $5 / 150$ & $-25 \%$ \\
\hline
\end{tabular}




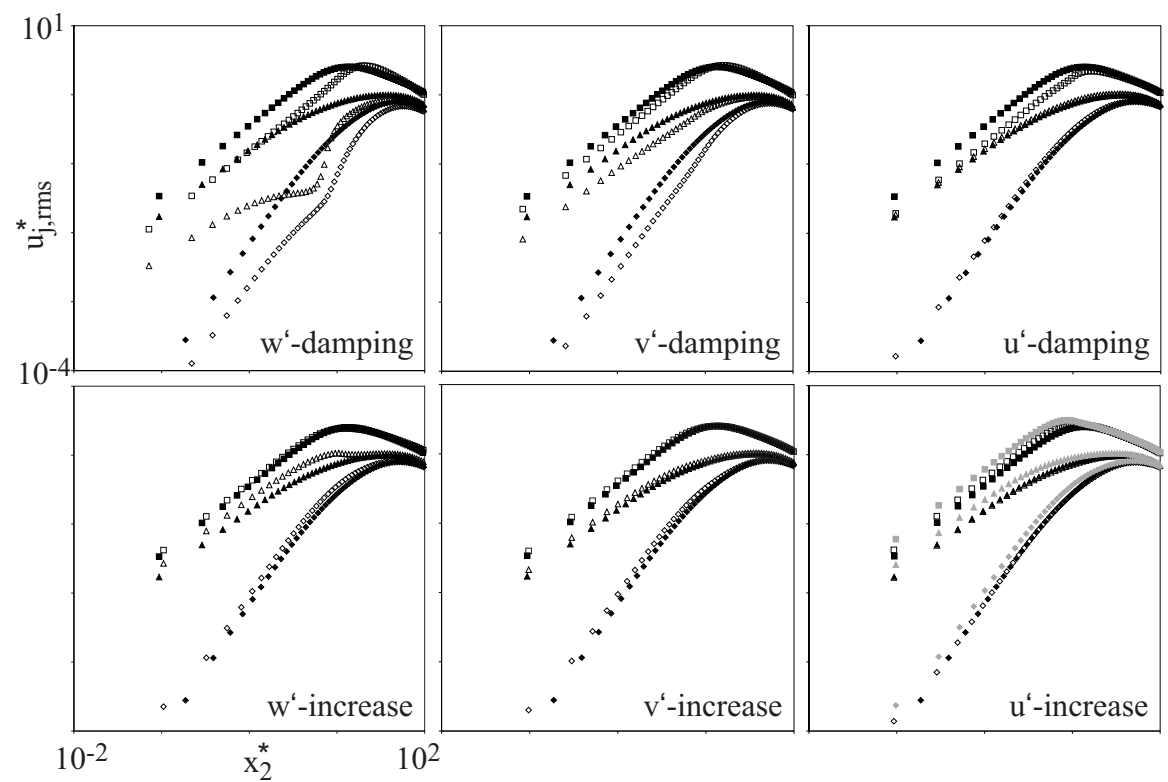

Fig. 1 Near-wall behavior of the rms values $\left(u_{r m s}^{*}, v_{r m s}^{*}, w_{r m s}^{*}\right.$ from top to bottom in each plot) for the controlled flow (open symbols) in comparison to the uncontrolled channel flow (solid symbols). The subscript ()$^{*}$ indicates that all values are normalized with the inner variables of the corresponding flow. The plot for u'-increase contains results for both, weak (black open symbols) and strong (grey solid symbols) forcing.

This amount of $D R$ can not be achieved with increasing the strength of v'-damping up to $\left(\Phi^{+}\right)^{-1}=-1000 / 15$, which corresponds to the maximum forcing strength considered in the present investigation.

For positive values of $b f_{2}$ and $b f_{3}$, which increase $v^{\prime}$ and $w^{\prime}$, respectively, drag increase is observed. Again, a body force acting on the $w$-component $\left(b f_{3}\right)$ has a more pronounced effect than $b f_{2}$ with similar values of $\left(\Phi^{+}\right)^{-1}$. The general similarity between modifications of the $v$ - and $w$-component can be explained as a result of streamwise vortices in the near-wall region which are damped or enhanced due to the introduced body forces.

For body forces acting in the streamwise direction, $b f_{1}$, drag increase is observed for damping of the streamwise fluctuations, $u^{\prime}$. This is in contrast to the results of [5] who reported slight $D R$ for an exponentially decaying damping force in streamwise direction. In the present investigation, weak enhancement of the streamwise velocity fluctuations leads to a slight $D R$, while stronger forcing enhances the skin friction drag. Figure 2 shows the near-wall behavior of the velocity fluctuations of the controlled flow in comparison to the uncontrolled channel flow. It can be seen that strong body forces in spanwise or wall-normal direction significantly modify the other near-wall components, too. 
Fig. 2 Anisotropy-invariant mapping for the uncontrolled flow and one of the controlled (w'-damping, $\mathrm{DR}=42 \%$ ) flows. The second invariant, $I I_{a}$, captures the magnitude of anisotropy and arrows mark the value of $I I_{a}$ at the wall.

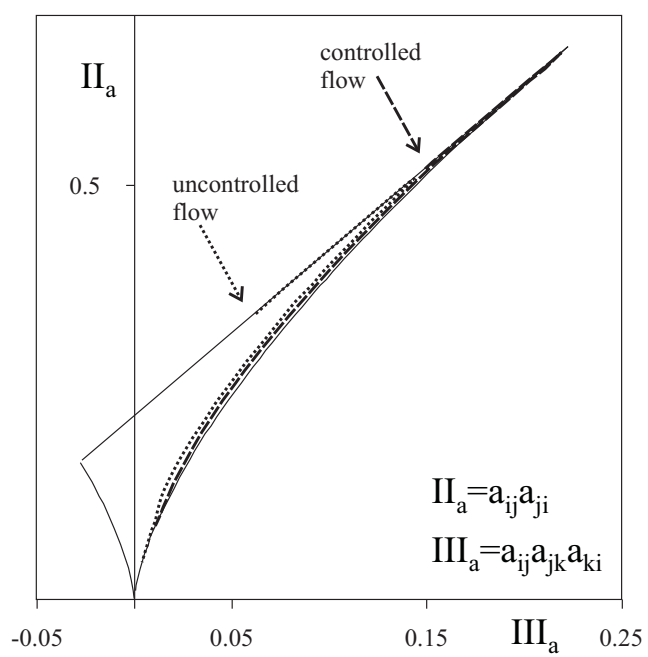

In [1] it was demonstrated that, for a variety of different flow control techniques, $D R$ is associated with increased anisotropy of turbulence in the near-wall region. When the near-wall behavior of the present controlled flows is analyzed in the anisotropy-invariant map (see figure 2), an increase of the second invariant, $I I_{a}$, of the anisotropy tensor, $a_{i j}[6]$ :

$$
a_{i j}=\frac{\overline{u_{i}^{\prime} u_{j}^{\prime}}}{q^{2}}-\frac{1}{3} \delta_{i j}, I I_{a}=a_{i j} a_{j i},
$$

in the near-wall region is observed for all drag reduced cases. This result is in agreement with the above mentioned prior findings. It is interesting to check whether a reversed trend in the near-wall turbulence anisotropy can be found for drag increased flows. In the present investigation, the anisotropy in the near-wall region is decreased for all drag increased flows, expect for the case of strong streamwise forcing for which a slight increase of $I I_{a}$ is observed.

In general, the magnitude changes of the near-wall anisotropy cannot be related quantitatively to the Reynolds shear stress, $-\overline{u^{\prime} v^{\prime}}$, which forms the turbulent contribution to the skin friction drag in a fully developed turbulent channel flow [3]:

$$
c_{f}=\frac{12}{R e_{b}}+12 \int_{0}^{1} 2(1-y)\left(-\overline{u^{\prime} v^{\prime}}\right) d y
$$

Further information about $-\overline{u^{\prime} v^{\prime}}$ can be found when considering the transformation of the Reynolds stress tensor to its diagonal form which forms the basis for the invariant analysis: 


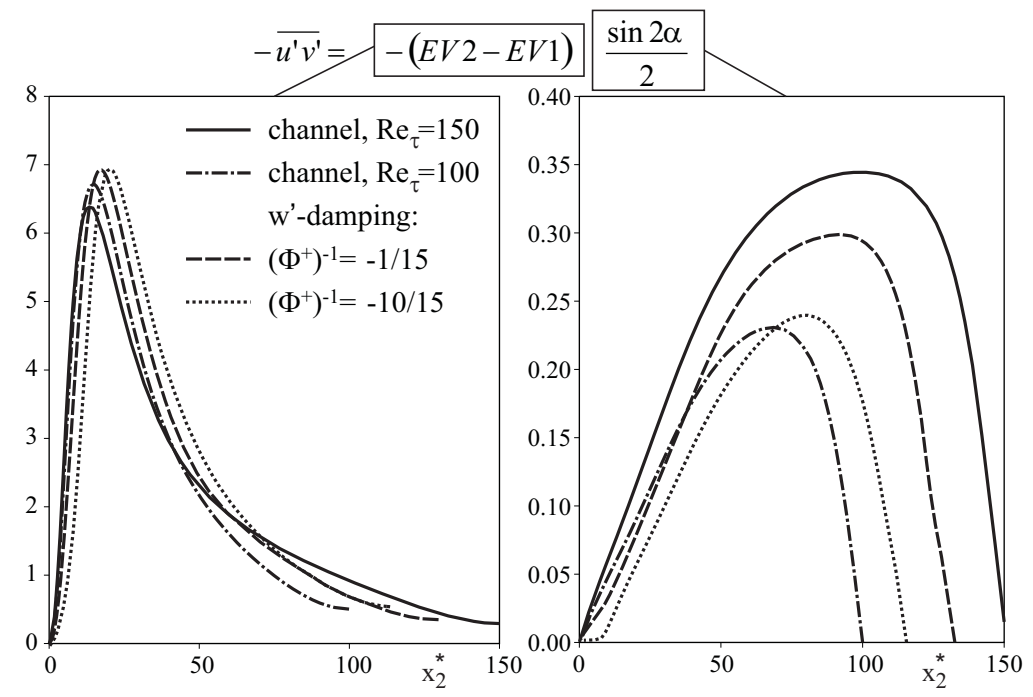

Fig. 3 Contributions to the Reynolds shear stress in terms of the eigenvalues of the Reynolds stress tensor and the misalignment of the principle axis with the channel coordinate system. Normalization is based on the inner variable of each flow.

$$
\left(\begin{array}{ccc}
\cos \alpha & \sin \alpha & 0 \\
-\sin \alpha & \cos \alpha & 0 \\
0 & 0 & 1
\end{array}\right)\left(\begin{array}{ccc}
\overline{u^{\prime} u^{\prime}} & \overline{u^{\prime} v^{\prime}} & 0 \\
\overline{u^{\prime} v^{\prime}} & \bar{v}^{\prime} v^{\prime} & 0 \\
0 & 0 & \overline{w^{\prime} w^{\prime}}
\end{array}\right)\left(\begin{array}{ccc}
\cos \alpha-\sin \alpha & 0 \\
\sin \alpha & \cos \alpha & 0 \\
0 & 0 & 1
\end{array}\right)=\left(\begin{array}{ccc}
E V 1 & 0 & 0 \\
0 & E V 2 & 0 \\
0 & 0 & E V 3
\end{array}\right) \text { (4) }
$$

It is possible to express $-\overline{u^{\prime} v^{\prime}}$ in terms of the angle $\alpha$ of this rotation and the difference of the first and second eigenvalues, $E V$, of the Reynolds tensor:

$$
-\overline{u^{\prime} v^{\prime}}=-(E V 2-E V 1) \frac{\sin 2 \alpha}{2} .
$$

Figure 3 shows the change in both terms of this identity for two $w^{\prime}$-damping cases in comparison with the uncontrolled channel flow. In order to distinguish between Reynolds number effects (for the controlled flows $R e_{\tau}=130$ and $R e_{\tau}=115$, respectively) and the influence of the applied control, channel flow data of $R e_{\tau}=100$ is also included. It is obvious that the reduction of the angle $\alpha$, i.e. the reduced misalignment between the principal axis and mean flow direction, in the near-wall region is the main reason for the reduction of the Reynolds shear stress and thus $D R$, while the reduction of $\alpha$ in the outer flow region can be attributed to the decrease in Reynolds number. The same trends are found for v'-damping. For weak u'-enhancement, only $D R=2.2 \%$ is achieved and the corresponding changes in the quantities of equation (5) are small but they suggest the same conclusion: the reduction of $\alpha$ can be identified as the source for the reduced Reynolds shear stress.

The drag increase for u'-damping, v'-increase and w'-increase is related to a general increase of $\alpha$ while $(E V 2-E V 1)$ is reduced. Especially for $u^{\prime}$-damping a 
drastic increase of $0.5 \sin 2 \alpha$ is observed in the forcing layer. It is accompanied by a strong increase of the correlation coefficient for $-\overline{u^{\prime} v^{\prime}}$ in this region. This fact explains the increase of the Reynolds shear stress in spite of damping of the streamwise velocity fluctuations $u^{\prime}$.

For strong enhancement of the streamwise velocity fluctuations, an increase of $I I_{a}$ is observed while the skin friction drag is increased. This result suggests that the commonly observed trend of increased near-wall anisotropy for drag reduced flows cannot be identified as a sufficient condition for $D R$. The analysis in terms of eigenvalues and alignment can shed some light into this point of discussion. Figure 4 shows the split-up of the corresponding Reynolds shear stress into its contributions according to equation (5). In the near-wall region a reduction of the $\alpha$-term in comparison to the uncontrolled channel flow can be observed. Although the Reynolds number effect would correspond to an increase of $\alpha$ in the entire flow domain, it remains identical to the one for an uncontrolled channel flow in the region of approximately $30<y^{+}<100$ before shifting to higher values around the channel center. In spite of the $\alpha$-reduction in the near-wall region $-\overline{u^{\prime} v^{\prime}}$ increases due to a large increase of $(E V 2-E V 1)$. This term can further be split up into the turbulent kinetic energy, $k=0.5 q^{2}$, of the flow field and the difference between first and second eigenvalue of the anisotropy tensor, $a_{i j}$, according to:

$$
(E V 2-E V 1)=q^{2}\left(E V 2\left(a_{i j}\right)-E V 1\left(a_{i j}\right)\right)
$$

The split up reveals that the large increase in the eigenvalue difference $(E V 2-E V 1)$ and thus the increase of $-\overline{u^{\prime} v^{\prime}}$ can be related to the increase of turbulent kinetic energy in the flow field due to the enhancement of $u^{\prime}$. This behavior suggests that the high turbulent kinetic energy, induced by the $u^{\prime}$-enhancing body force, suppresses the drag reducing potential of the improved alignment in the near-wall region. In this respect it should be noted that the energy input required to change $v^{\prime}$ and $w^{\prime}$ is generally small compared to the pumping power of the channel flow while significantly higher energy input is needed for changes of $u^{\prime}$.

In summary, the results of the present investigation suggest that flow control that acts on the w'-component is most effective. Modifications of the v'-component yield similar results but tend to be less effective resulting in lower gain, i.e. energy savings per energy input for the $D R$ cases. Direct modifications of the streamwise velocity fluctuation yield the surprising result that drag increase is found for a reduction of the turbulent kinetic energy in the flow field and vice versa (for small increase of $u^{\prime}$ ). Since these modifications require high energy inputs, however, they do not seem a smart target for flow control. The misalignment angle, $\alpha$, between principle axis of the Reynolds stress tensor and the mean flow direction of the channel is introduced as an interesting quantity for flow control. At this point the present results suggest that a reduction of $\alpha$ in the near-wall region which is achieved with little energy input will result in drag reduction. Much further work will be needed to clarify whether this fact can be used in the design of practical flow control techniques. Simulations of $w^{\prime}$-damping at $R e_{\tau}=300$ and 450 show that the drag reduction rate achievable with near-wall componental modifications exhibits almost no Reynolds 


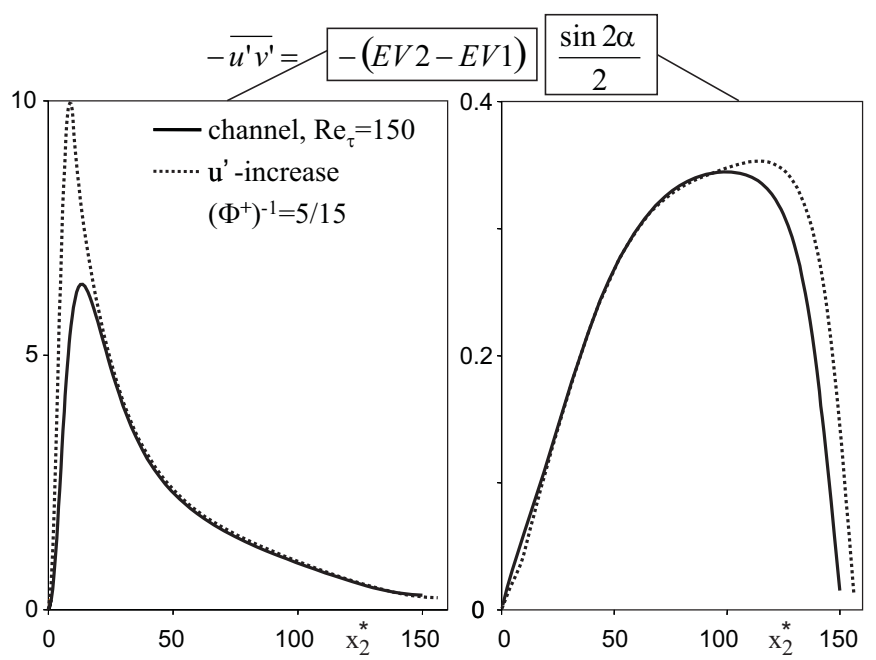

Fig. 4 Contributions to the Reynolds shear stress in terms of the eigenvalues of the Reynolds stress tensor and the misalignment of the principle axis with the channel coordinate system. Normalization is based on the inner variable of each flow.

number dependency, at least in the investigated regime, and confirm that the reduction of the misalignment angle $\alpha$ is a key quantity for the reduction of the Reynolds shear stress, $-\overline{u^{\prime} v^{\prime}}$.

\section{References}

1. Frohnapfel B, Hasegawa Y, Kasagi N (2008) Flow Control by Turbulence State Modifications in the Near-Wall Region. In: XXII Int Cong Theoretical and Applied Mechanics, Adelaide, Australia

2. Frohnapfel B, Lammers P, Jovanović J, Durst F (2007) Interpretation of the mechanism associated with turbulent drag reduction in terms of anisotropy invariants. In: J Fluid Mech 577: 457-466

3. Fukagata K, Iwamoto K, Kasagi N (2002) Contribution of Reynolds stress distribution to the skin friction in wall-bounded flows. In: Phys. Fluids 14: L73-L76

4. Iwamoto K, Fukagata K, Kasagi N, Suzuki Y (2005) Friction drag reduction achievable by near-wall turbulence manipulation at high Reynolds number. In: Phys Fluids 17: 011702

5. Lee C, Kim J (2002) Control of the viscous sublayer for drag reduction. In: Phys Fluids 14: 2523-2529

6. Lumley JL, Newman GR (1977) The return to isotropy of homogeneous turbulence. In: J Fluid Mech 82: 161-178 